Ewa Stala

Universidad Jaguelónica

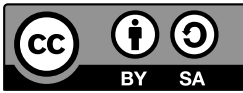

\title{
El idioma de los argentinos: entre Borges y Bordelois
}

Palabras clave: español de Argentina, idioma nacional, ecolingüística, política lingüística, higiene verbal

\section{Introducción}

La inspiración de esta ponencia surgió del título de la conferencia (Entre Cervantes y Borges. Perspectivas y desafíos organizada en la Facultad de Filosofía y Letras de la Universidad de Ljubljana del 3 al 5 de noviembre de 2016), inquietudes anteriores (Stala, 2004, 2009, en prensa) y la lectura de otros trabajos (Agoff et al., 2013; Thon, 2010; di Tullio, 2011; Leśniak, 2014) sobre el español de la Argentina. Nuestra intención ha sido presentar las ideas principales acerca de esta variedad del idioma en su dimensión diacrónica: la idea ya histórica de Jorge Luis Borges y la contemporánea, de Ivonne Bordelois. Asimismo, estas divagaciones nos acercan a las preguntas de siempre: ¿qué es el idioma nacional? ¿para qué sirve? ¿qué lo ayuda y qué obstaculiza su desarrollo?

\section{Base teórica}

La base teórica de este artículo la constituyen los conceptos relacionados con la ecología de la lengua o ecolingüística o sea la interacción entre la lengua y el medioambiente ( $c f$. Haugen, 1972: 6 et passim), política lingüística comprendida como conjunto de ideas que están creando a su vez la planificación lingüística, donde esta última abarca la ciencia que tiene por objetivo influir en el uso de una lengua concreta, su difusión, estatus social y sus funciones ${ }^{1}$. Porque, tal

1 Ver por ejemplo, la Carta Europea de las Lenguas Minoritarias y Regionales, promulgada por la Comunidad Europea. 
como dijo Copper (en Leśniak, 2014: 8): «Like cities, language structure and use are subject to planning and, like cities, they change whether planned or not». Esta observación presenta el cambio lingüístico como un proceso natural de la lengua y la planificación lingüística como una actividad natural para el ser humano. A su vez Haugen (1987: 19 et passim) diferencia entre la planificación de estatus (a nivel de la sociedad) y la planificación de corpus (a nivel de la lengua). Y hace diferencia entre la planificación y la cultivación. Así, primero sucede la selección (de una lengua o variante), luego la codificación (estandarización), implementación (difusión educativa) y, al final, la elaboración (desarrollo funcional o adaptación a nuevos usos). En este contexto un término muy útil es el de verbal bygiene, acuñado por Deborah Cameron en su libro de 2005. La autora, al rechazar la oposición entre descriptivismo-prescriptivismo y al evitar la asociación negativa del segundo, propone el término verbal bygiene como toda reflexión metalingüística que se da a diario entre los hablantes por el carácter social de la lengua. De hecho, la cultivan tanto Borges como Bordelois en sus obras dedicadas al idioma de los argentinos.

\section{Política lingüística del español - esbozo histórico}

Si, siguiendo a Furdal (1990: 248), definimos la nación como un grupo social que se sirve de una lengua común para comunicarse y distinguirse de otros grupos, la lengua se ve como uno de los rasgos distnitivos, al lado de la religión o la historia o la economía, de una sociedad. De esta manera, la lengua es una de las fuentes del sentimiento gregario nacional. En caso del español, indudablemente, los primeros en ocuparse de la política lingüística fueron: Alfonso X el Sabio y, a continuación, los Reyes Católicos. Es cuando se produce la unificación nacional y cuando surge la primera gramática y el primer vocabulario de la lengua castellana. Es en el prólogo de la Gramática donde el autor, E. A. de Nebrija nota:

Cuando bien comigo pienso, mui esclarecida Reina, i pongo delante los ojos el antigüedad de todas las cosas, que para nuestra recordación y memoria quedaron escriptas, una cosa hállo y: sáco por conclusión mui cierta: que siempre la lengua fue compañera del imperio; y de tal manera lo siguió, que junta mente començaron, crecieron y florecieron, y después junta fue la caida de entrambos (Nebrija, 2012: 11).

La Real Academia Española se funda en 1713. En 1715 se elige el lema de la Academia: «Limpia, fija y da esplendor». Aunque casi inmediatamente surgen 
las primeras obras (Diccionario 1726-1739, Ortografía 1741, Gramática 1771), el siglo XIX ya no es tan exitoso (Lapesa, 1987: 335). Si bien a pocos años de su fundación la Academia invita a los intelectuales hispanoamericanos a colaborar con ella, una vez lograda la independencia política, los invitados no quieren volver a subordinarse a España, también en el sentido meramente lingüístico (López Morales, 2005: 922). Así, en 1871 nace la Academia Colombiana y le siguen las otras, formando, de este modo, un paso importante en la emancipación lingüística de los países hispanohablantes. El congreso de la Asociación de las Academias de Lengua Española, de 1950, organizado en México, dio comienzo a un nuevo tipo de colaboración y la política lingüística actual: la llamada política panbispánica ${ }^{2}$. El primer fruto sale en 1999: es la nueva edición de la Ortografía de la lengua española a la que siguen la $22^{\mathrm{a}}$ edición del Diccionario de la lengua española en 2001 y la Nueva gramática de la lengua española en 2009.

\section{Situación lingüística en Argentina}

En caso de la Argentina, los miembros de la llamada generación del 37 (Juan Bautista Alberdi, Domingo Faustino Sarmiento, Juan María Gutiérrez) forman su Salón Literario en 1837. Como admite Leśniak (2014: 25), «lo característico de la generación del 37 es el significado de la noción del idioma nacional o bien idioma argentino». Pero cuando llega el Centenario (1910), el país enfrenta un nuevo problema: a causa de las oleadas inmigratorias, se ve indispensable la búsqueda de la identidad nacional y, por consiguiente, la redefinición de las posturas hacia la lengua nacional. Así se forman dos actitudes: la casticista e hispanófila y la liberal, de inclusión y búsqueda de rasgos propios. Es cuando surge la Academia Argentina de Ciencias y Letras que opera entre 1873-1879 (Barcia, 2002) y en 1931 se forma la Academia Argentina de Letras cuyos propósitos principales son: el estudio especializado de las peculiaridades de la variedad argentina y el fomento de la literatura nacional y su conocimiento entre los mismos argentinos. Uno de sus últimos frutos es el Diccionario del Habla de los Argentinos de 2003 (la segunda edición es de 2008 y en 2017 está por aparecer las tercera).

La polémica acerca del idioma de los argentinos fue iniciada por Lucien Abeille ya en 1900 con su obra El idioma nacional de los argentinos, publicada en París, cuya tesis rupturista abre de este modo el debate conocido como «cuestión del idioma» (di Tullio, 2011: 191). Cuando en Buenos Aires, en 1922 se fundó el

2 http://www.asale.org/la-asociacion/politica-linguistica-panhispanica/hechos-relevantes 
Instituto de Filología, cuyos directores fueron, entre otros, Américo Castro (1924-26) y Amado Alonso (1928-45), la variedad argentina se ve como una peculiaridad o un problema ${ }^{3}$. Semejante postura produce rechazo de parte de los intelectuales, entre ellos, J. L. Borges.

\subsection{La óptica borgeana}

Las ideas borgeanas se crean en las primeras décadas del siglo XX, cuando, tras haber logrado la independencia política, se disputa la independencia intelectual y lingüística, mientras aumenta la inmigración masiva desde Europa. Entonces, por un lado se objeta el academismo español y, acorde el romaniticismo de la época, se nota la mayor aceptación de rasgos populares y por el otro, se ve amenazada la homogeneidad lingüística, más cercana al nacionalismo. A todo ello hay que agregar la presencia de Borges en el grupo de Florida, centrado alrededor del periódico literario Martín Fierro (publicado en 1924-37) con su ideario vanguardista.

El primer texto en el que aparece el pensamiento borgeano sobre el idioma es el Prólogo en su segundo tomo de poesía Luna de enfrente (1925), suprimido en las ediciones siguientes. Ahí dice:

El idioma se suelta. Los verbos intransitivos se hacen activos y el adjetivo sienta plaza de nombre. Medran el barbarismo, el neologismo, las palabras arcaicas. Frente al provincionalismo remilgado que ejerce la Academia [...] nuestro idioma va adinerándose. No es de alto ríos soslayar la impureza, sino aceptarla y convertirla en su envión (Borges, 1997: 198).

Es cuando se aclaran sus dos tendencias principales: la crítica de la Academia y la apertura a las creaciones nuevas. Pero también la supremacía del porteño: frente a lo regional (provincia argentina) y lo externo (la masa inmigratoria). Como dicen di Tullio y Bordelois (2002): «Sólo los porteños de rancia prosapia ejercen naturalmente esta norma, que excluye a los españoles autoritarios, a los provincianos reaccionarios y a los italianos advenedizos»4.

En 1926 Borges publica el segundo tomo de sus ensayos El tamaño de mi esperan$z a$ y ahí figuran los textos que explícitamente hablan de sus ideas lingüísticas:

3 Cf. La peculiaridad lingüistica rioplatense y su sentido bistórico de A. Castro (1941) o «E1 problema argentino de la lengua», el primer capítulo de El problema de la lengua en América (1935) de A. Alonso.

4 http://www.lehman.cuny.edu/ciberletras/vo6/bordelois.html 
El idioma infinito, Palabrería para versos e Invectiva contra el arrabalero. En los dos primeros describe las posibilidades del desarrollo del léxico, para que el lenguaje común pueda llegar a crear el poético y ridiculiza la postura académica acerca del uso correcto de la lengua: más vale encontrar el lenguaje preciso que aplicar varios sinónimos: lo primero es «riqueza esencial» y lo segundo «aparencial» (Borges, 1998b: 43). También rechaza tanto los seguidores de la cultura francesa, como la netamente española: «Dos conductas de idioma [...] se dan en esta tierra: una la de los haraganes galicistas que a la rutina quieren anteponer otra rutina [...], otra, la de los casticistas, que creen en la Academia como quien cree en la Santa Federación y a cuyo juicio ya es perfecto el lenguaje.» (Borges, 1998b: 42).

En el tercero, define el lunfardo como «la tecnología de furca y ganzúa», «una jerga artificiosa de los ladrones» y el arrabalero como «la simulación de esa jerga» (Borges, 1998b: 158-159). Critica su pobreza del léxico, su poco valor estético, su dudoso estatus moral. Y propone una solución para hacerlo ascender a la dignidad de lengua: «la ciudad sigue a la espera de una poetización» (Borges, 1998b: 168), es decir, el lenguaje debe hacerse una herramienta literaria. Es la clara continuación de la filolosofía de B. Croce, presentada a lo largo de su libro Estética como ciencia de la expresión y lingüística generals, quien identifica la lengua con su expresión poética y literaria.

Pero el ensayo más conocido que trata de las cuestiones lingüísticas es, por supuesto, El idioma de los argentinos publicado en 1927. En él, según dicen sus investigadoras, di Tullio y Bordelois (2002), Borges aboga que «en materia de autenticidad lingüística, la intuición nativa y la estética propia valen más que los fatigosos y fatigantes estudios de los universitarios» ${ }^{6}$. Ahí expresa su actitutd crítica frente al arrabalero por su pobreza de léxico, poco uso en la sociedad y escasez en la literatura ( de ahí la preferencia de los primeros tangos donde aún no reinaba el lunfardo) y censura el purismo lingüístico, comúnmente asociado con la Academia. Otra vez, contrasta la riqueza cualitativa con la cuantitativa: «La riqueza del español es el otro nombre de su muerte. Abre el patán y el que no es patán nuestro diccionario y se queda maravillado frente al sinfín de voces que están en él y que no están en ninguna boca» (Borges, 1998a: 150). Con estas palabras reitera la crítica de la sinonimia y el elogio de la oralidad. A su vez aboga tanto por la unidad del idioma como por su carácter propio: «¿Qué zanja insuperable hay entre el español de los españoles y el de

5 Manejamos la edición española de 1997. La obra fue publicada originalmente en 1902 y traducida al español en 1912.

6 http://www.lehman.cuny.edu/ciberletras/vo6/bordelois.html 
nuestra conversación argentina? [...] ninguna, venturosamente para la entendibilidad general de nuestro decir» (Borges, 1998a: 156). Para él, el idioma argentino es «una forma argentina de expresarse en español» y sigue:

Pienso en el ambiente distinto de nuestra voz, en la valoración irónica o cariñosa que damos a determinadas palabras, en su temperatura no igual. No hemos variado el sentido intrínseco de las palabras, pero sí su connotación. Esa divergencia, nula en la prosa argumentativa o en la didáctica, es grande en lo que mira a las emociones. Nuestra discusión será hispana, pero nuestro verso, nuestro humorismo, ya son de aquí (Borges, 1998a: 156).

Sin embargo, el fragmento crucial se encuentra al final de la publicación mencionada, El idioma de los argentinos:

Pero nosotros quisiéramos un español dócil y venturoso, que se llevara bien con la apasionada condición de nuestros ponientes y con la infinitud de dulzura de nuestros barrios y con el poderío de nuestros veranos y nuestras lluvias y con nuestra pública fe. Sustancia de las cosas que se esperan, demostración de cosas no vistas, definió San Pablo la fe. Recuerdo que nos viene del porvenir, traduciría yo. La esperanza es amiga nuestra y esa plena entonación argentina del castellano es una de las confirmaciones de que nos habla (Borges, 1998a: 160).

Finalmente, el texto más acérrimo de Borges fue la respuesta ofrecida al director del Instituto de Filología, Américo Castro, quien en su ensayo La peculiaridad rioplatense y su sentido bistórico de 1941 critica el «desbarajuste lingüístico», pero más aun la «impunidad social» de las incorecciones cometidas. Acusa a los argentinos de una «anárquica espontaneidad» y de la «rebeldía de voseo». Borges en su ensayo Las alarmas del doctor Américo Castro no solo aplica argumentos sólidos sino arrasa a su contrincante con su brillante estilo literario. Así critica no solo sus ideas sino su estilo: «En la página 122, el doctor Castro ha enumerado algunos escritores cuyo estilo es correcto; a pesar de la inclusión de mi nombre en este catálogo, no me creo del todo incapacitado para hablar de estilísitca.» (Borges, 2008: 60) para luego agregar: «[Castro] acumula retazos de Pacheco, de Vacarezza, de Lima, de Last Reason, de Contursi, de Enrique González Tuñón, de Palermo, de Llanderas, de Malfatti, los copia con inútil gravedad y luego los exhibe, urbi et orbi como ejemplos de nuestro 
depravado lenguaje.» (Borges, 2008: 57) y proceder con la crítica del español de los españoles: «confunden acusativo y dativo, dicen le mató por lo mató, suelen ser incapaces de pronunciar Atlántico y Madrid, piensan que un libro puede sobrellevar este cacofónico título: La peculiaridad rioplatense y su sentido bistórico» (Borges, 2008: 59).

Borges se distancia tanto de las posturas cultas y encerradas como del lenguaje plebeyo o avulgarado. Lo que le interesa es la aparición de un lenguaje culto de Buenos Aires: propio pero con rasgos elevados, el que está por hacerse":

La solución de Borges a la cuestión del idioma no consiste en recurrir a España, como hizo el Centenario, ni acomodarse bajo la protección de otro idioma extranjero, sino que invirtió las jerarquías establecidas para proponer una nueva: el sector intelectual de la Argentina al que pertenece está en condiciones de ejercer el liderazgo para convertir a su modalidad lingüística en el marco de referencia de su comunidad, entendida en sentido más o menos amplio (di Tullio, 2011: 204).

\subsection{El idioma argentino según Bordelois}

Ivonne Bordelois, eminente lingüista nacida 35 años más tarde que Borges, pertenece a una nueva época. Ambos comparten la estadía fuera del país natal ${ }^{8}$, ambos también se desempeñaron en la literatura99.

En su libro de la serie lingüística, La palabra amenazada, del año 2003, propone una estrategia ecológica: «explorar cuáles son las maneras no violentas de la celebración del lenguaje entre nosotros. Es decir, explorar cuáles son las maneras de recuperación y escuchar del lenguaje que nos lo vuelvan al íntimo, viviente y disfrutable» (Bordelois, 2005: 37) ${ }^{10}$.

La pregunta que surge automáticamente es: ¿de qué violencia rescatar la lengua? y ¿qué es lo que amenaza a la palabra? La respuesta la ofrece ya al principio de su libro: «he tratado de bosquejar una estrategia para el rescate de la palabra en el mundo contemporáneo» en los tiempos en los que la conciencia

7 Por razones de espacio hemos omitido tales cuestiones como el proyecto de la reforma ortográfica de Borges o su libro escrito con J. Clemente (1963): El lenguaje de Buenos Aires.

8 En caso de Bordelois, causada por la dictadura ( $c f$. di Tullio 2007: 133).

9 Las obras literarias más destacadas de Bordelois son: El alegre apocalipsis de 1995, Correspondencia Pizarnik de 1998, Un triángulo crucial: Borges, Lugones y Güiraldes, 1999.

10 Manejamos la edición de 2005. 
lingüística queda cada vez más aniquilada por la «esclavitud laboral, informática y consumista» (Bordelois, 2005: 9). Según Bordelois, la primera violencia que experimenta la lengua es ser tratada como mero medio de comunicación y postula tratarla como «órgano de conocimiento anterior al pensamiento» (2005: 13) lo que inmediatamente implica su aplicación en literatura y, sobre todo, poesía. Lo mismo que años atrás propuso Borges. También propone cambiar el signo de globalización por el de la analogía: «una operación por medio de la cual, gracias al juego de las semejanzas, aceptamos las diferencias. La analogía, ciencia de las correspondencias, no las suprime: las redime, implicando, no la unidad del mundo, sino su pluralidad» (2005: 101). Pero Bordelois no solamente se refiere a los poetas de renombre, sino también a los creadores diarios de la lengua. Por eso queda maravillada con algunos giros y expresiones escuchadas en la calle o en la televisión (sic!): decir jbárbaro ; ; brutal! o lo más poético ijoya! para expresar la admiración; no bay drama en vez de no problem, el vesre lunfardesco con todo lo que implica («no es lo mismo telo que botel, rope que perro, chochamu que muchachos», 2005: 75) o hasta los letreros en las paredes de Palermo Viejo ${ }^{11}$ : «Démonos una mano, dijo la Venus de Milo» o «Anoche soñé con Dios. Pero yo no lo maté» para terminar con una observación muy ecológica:

El lenguaje es un fermento indestructible de unidad y comunidad entre nosotros - acaso uno de los últimos que quedan. Es el primer basamento, el estrato profundo en que se encuentra y se alimenta una comunidad: no contaminemos el agua de la que bede nuestra vida, no la dejemos a merced de los mercaderes de excrementos (Bordelois, 2005: 136).

Pareciera que los dos hablan diferente porque pertenecen a dos épocas diferentes: Borges se preocupa por la constitución del idioma de los argentinos porque lo ve formarse, Bordelois ya lo ve como un ente establecido, solo sugiere cuidarlo y cultivarlo. Pero dos años más tarde, en 2005, publica otro libro-ensayo dedicado al tema de la lengua, El país que nos babla ${ }^{12}$ y ahí vuelve a la cuestión de lo argentino. Partiendo de la tesis de que «toda nación se constituye alrededor de su lenguaje: plural o único, dominante o dominado, imperial o colonizado» (Bordelois, 2006: 29) ${ }^{13}$, la autora presenta una breve historia del argentino como idioma nacional (2006: 45-70) hasta llegar a las postura del mismo... Borges. Y en seguida enumera las amenazas actuales:

11 Un barrio muy pintoresco de Buenos Aires.

12 Por el que ese mismo año recibe el Premio La Nación-Sudamericana.

13 Manejamos la edición de 2006. 
Hasta aquí Borges. Hoy podríamos reescribir estas líneas con leves variaciones. No es ya la norma hispánica obsoleta la que nos desfigura, sino la apetencia de parecer globales y actualizados y hablar de sales en vez de saldos, o bien la de adoptar una chabacanería ilimitada que acaba por convertir es un depósito de basura verbal los programas de televisión más exitosos, las letras de canciones más repetidas o las páginas más socorridas de las revistas amarillas de todo tipo (Bordelois, 2006: 70).

Es donde se ven claramente las «alarmas» de Bordelois: la creciente invasión de los anglicismos hasta llegar al mestizaje pleno, en caso del Spanglish, la obligación constante de «allanar» el español para poder ser difundido en todo el continente o sea que «nos obliguen imperialmente al tuteo, a las cerillas, a las piscinas y a los grifos» ${ }^{14}$. Pero igual le preocupa el empobrecimiento del léxico entre la población juvenil ya que «si no hay palabras, el único vehículo accesible para desalojar la fuerza de las pasiones interiores es la violencia» (2005: 123) o la reducción de lenguaje en el chat que «no sólo chatea sino que achata» aunque requiere y suscita mucha creatividad de parte de los usuarios. El caso opuesto del centrípeto chat es el centrífugo lunfardo que «nos retrotrae a las coloridas raíces cosmopolitas de nuestro país» (2005: 135) y aunque se originó como «un dialecto de resistencia social contra el habla culta de las clases altas» (de ahí la poca simpatía de parte de Borges), acabó por desprenderse en la sociedad, creando su propia estética y literatura ${ }^{15}$.

Bordelois también divisa el elemento ingenioso de los usuarios de la variedad argentina, «chispazos de humor, ingenio o malicia» (2005: 139). Las creaciones tipo sip, nop, porsu, tranqui, el muy productivo prefijo re-, los derivados tipo flashear 'deslumbrarse', filtrado 'cansado', la curiosa trayectoria de la voz quilombo de 'lugar fortificado o secreto' a 'situación complicada' o los neologismos creados durante la crisis económica: cartonero, menemtrucho, corralito, piqueduro, piqueblando. Entonces, tal vez, ¿para rescatar la palabra solo alcanza con canalizar la creatividad de los usuarios? Porque si bien Bordelois coincide con Borges en la «libertad nacional» de la lengua o sea su independencia de las reglas académicas peninsulares y en la necesidad de la creación culta, no rechaza la creatividad cotidiana, el despreciado por Borges elemento arrabalero y lunfardo, más bien lo ve como una emanación de la poesía popular, la creatividad de idioma necesaria para luego transponerlo a las alturas del arte

14 En este fragmento Bordelois se refiere, por supuesto, al voseo rioplatense y los equivalentes léxicos: fósforos, pileta, canilla.

15 El mejor ejemplo son las numerosas e ingeniosas letras de tango. 
poético ya que: «El lenguaje es el depósito sagrado de nuestra conciencia, la condición de nuestra sabiduría, la garantía de nuestra identidad y de nuestra libertad, y también una fuente de placer inagotable, si sabemos encontrarla» (Bordelois, 2006: 218).

\section{$5 \quad$ A modo de conclusión}

La mencionada al principio de este artículo Irene Agoff junto con otros coautores en su artículo publicado en el diario Página 12, indica:

Borges es el Cervantes del siglo XX: ésto es, el renovador mayor de la lengua, no sólo para su país natal sino para el conjunto de los hispanohablantes. Si en los años veinte buscó en la sonoridad de la criolledá la expresión idiomática propia, una década después descubría que no se trata de color local: que la lengua estaba en un tono, una respiración, una andadura. Lo hizo de modo polémicos y no poco cuestionables, como su carácter antiplebeyo y sus derivas conservadoras. Pero es el momento de recuperar con su nombre, una apuesta que toma la suya como inspiración y al mismo tiempo debe modificarla (Agoff et al., 2013: 4-5).

Se puede decir, aplicando los términos de Haugen, que si bien, por razón de la época, Borges opera en los cuatro niveles (selección, codificación, implementación y elaboración), Bordelois se limita a los últimos tres. Bordelois tampoco se opone a lo plebeyo, es más: nota su valor y su ingenio. Pero igual aboga por el rescate de la lengua en el sentido de cuidarla, enriquecerla, cultivarla. Ambos subrayan el papel del lenguaje literario y sobre todo el poético como un medio de rescate de la lengua nacional. En fin, la visión de ambos difiere: una más clasista, otra más democrática, pero en ambos se nota una gran preocupación por su lengua y su futuro. Tal como dijo el Maestro: «el porvenir [...] tira de nuestros corazones» (Borges, 1998a: 161).

\section{Bibliografía}

\section{Relación de fuentes}

Bordelois, I. ([2003] 2005): La palabra amenazada. Buenos Aires: Libros del Zorzal.

Bordelois, I. ([2005] 2006): El país que nos babla. Buenos Aires: Editorial Sudamericana. 
Borges, J. L. (1997): Textos recobrados 1919-1929. Buenos Aires: Emecé.

Borges, J. L. ([1928] 1998a): El idioma de los argentinos. Madrid: Alianza Editorial.

Borges, J. L. ([1928] 1998b): El tamaño de mi esperanza. Madrid: Alianza Editorial.

Borges, J. L. ([1925] 2008): Inquisiciones. Madrid: Alianza Editorial.

\section{Relación de estudios}

Abeille, L. (1900), El idioma nacional de los argentinos. Paris: E. Bouillon.

Alonso, A. (1935): El problema de la lengua en América. Madrid: Espasa-Calpe.

Barcia, P. L. (2002): «Brevísima historia de la Academia Argentina de Letras». Boletín de la Academia Argentina de Letras, 67, nº 263-264, 9-30.

Cameron, D. (2005): Verbal Hygiene. London-New York: Routledge.

Castro, A. (1941): La peculiaridad rioplatense y su sentido bistórico. Buenos Aires: Editorial Losada.

Clemente, J. (1963): El lenguaje de Buenos Aires. Buenos Aires: Emecé.

Croce, B. (1997): Estética como ciencia de la expresión y lingüistica general. Málaga: Ágora (trad. J. García Gabaldón, P. Aullón de Haro).

Diccionario del Habla de los Argentinos, 2003. Buenos Aires: Emecé Editores.

Furdal, A. (1990): Jezykoznawstwo otwarte. Wrocław: Zakład Narodowy im. Ossolińskich.

Haugen, E. (1972): The Ecology of Language. Language science and national development. Stanford: Stanford University Press.

Haugen, E. (1987): Blessings of Babel. Bilingualism and Language Planning. BerlinNew York-Amsterdam: Mouton de Gruyter.

Lapesa, R. (1987): Historia de la lengua española. Madrid: Gredos.

Leśniak, A. (2014): Lengua y nación según Jorge Luis Borges, tesis de licenciatura inédita. Universidad Jaguelónica.

López Morales, H. (2005): «La actuación de las academias en la historia del idioma». En: Rafael Cano (coord.), Historia de la lengua española. Barcelona: Ariel.

Nebrija de, E. A. ([1495?] 2012): Gramática de la lengua castellana. Barcelona: Red Ediciones.

Stala, E. (2015): «Las alarmas de Ivonne Bordelois: sinopsis del argentino contemporáneo». En: Eva Reichwalderová (ed.), Lengua, cultura y literatura en el contexto espacial y temporal, 168-178. 
Stala, E. (2004): «El castellano de Buenos Aires: entre Europa y América». En: Christian Wenztlaff-Eggebert (ed.), Europa como espacio cultural: Entre progreso y destrucción. Colonia: Universidad de Colonia, 188-192.

Stala, E. (2009): «Hiszpański Buenos Aires w kontekście historycznokulturowym». Studia Iberystyczne, 8, 103-110.

Thon, S. (2010): «La identidad lingüística argentina a través de Borges y Püig». ARBOR Ciencia, Pensamiento y Cultura, CLXXXVI, 741, 119-127.

Tulllio di, Á. (2011): «Borges y Arlt. Dos definiciones del idioma de los argentinos». En: Angela di Tullio, Rolf Kailuweit (eds.), El español rioplatense: lengua, literatura, expresiones culturales. Madrid/Frankfurt am Main: Iberoamericana/Vervuert.

\section{Enlaces de Internet}

Agoff, I. et al. (2013): «Por una soberanía idiomática»: http://www.pagina12. com.ar/diario/elpais/1-229172-2013-09-17.html (20-08-2016).

Bordelois, I., Tullio di, A. (2002): «El Idioma de los Argentinos: Cultura y Discriminación», Ciberletras: Revista de crítica literaria y de cultura, ISSN-e 1523-1720, 6: http://www.lehman.cuny.edu/ciberletras/vo6/bordelois.html (08-09-2016).

Tullio di, A. (2007): «La lingüística en la Argentina: una mirada retrospectiva»: https://dialnet.unirioja.es/servlet/articulo;jsessionid=C173 $\mathrm{D}_{95} \mathrm{C}_{516} \mathrm{D}_{34}$ ${ }_{63} \mathrm{~A}_{7} \mathrm{~B}_{1} \mathrm{C}_{79381 \mathrm{BA}}$ 739.dialneto1? codigo=2348685 (10-10-2016).

http://www.asale.org/la-asociacion/politica-linguistica-panhispanica/ hechos-relevantes (10-09-2016). 
Ewa Stala

Jagiellonian University

\section{The language of the Argentines: between Borges and Bordelois}

Keywords: Spanish of Argentina, National Language, Ecolinguistics, Linguistic Policy, Verbal Hygiene

This article presents two visions of the Argentinian national language: one of the emblematic and historical figure of the Argentinian literature, Jorge Luis Borges, the other, more contemporary, of the Argentinian linguist, poet and essay writer Ivonne Bordelois. Beyond the remarkable coincidences and differences highlighted not only by intellectual but also historical questions, the comparison is also a source of controversy over the character, form and role of any national language. The theoretical basis of this work are the ideas of Einar Haugen, i.e. the ecology of language, linguistic planning and linguistic policy. 


\section{Ewa Stala}

Jagelonska univerza

\section{Jezik Argentincev: med Borgesom in Bordeloisem}

Ključne besede: argentinska španščina, nacionalni jezik, ekolingvistika, jezikovna politika, govorna higiena

Pričujoči članek oriše dva pogleda na nacionalni jezik Argentincev: pogled emblematične in zgodovinske figure argentinske književnosti, Jorgeja Luisa Borgesa, in sodobnejši pogled argentinske jezikoslovke, pesnice in esejistke Ivonne Bordelois. Poleg očitnih sovpadanj in razhajanj, ki so plod ne le intelektualnih, temveč tudi diahroničnih vprašanj, primerjava spodbudi k problematizaciji značaja, oblike in vloge kateregakoli nacionalnega jezika. Teoretski temelji prispevka so osnovani na idejah Einarja Haugna: ekologija jezika, jezikovno načrtovanje in jezikovna politika. 